\title{
Estilos de aprendizaje, elección de carrera y perfil curricular en estudiantes de Comunicación Humana
}

\author{
Learning Styles, Degree Choice, and Curricular Profile in Human Communication Students \\ Estilos de aprendizagem, escolha de carreira e perfil de currículo em estudantes de \\ Comunicação Humana
}

Fernanda Gabriela Martínez-Flores* (http://orcid.org/0000-0002-2974-0876)

Facultad de Comunicación Humana, Universidad Autónoma del Estado de Morelos, Morelos, México. Ulises Delgado-Sánchez** (https://orcid.org/0000-0002-4318-0238)

Centro de Investigación Transdisciplinar en Psicología, Universidad Autónoma del Estado de Morelos, Morelos, México.
Recibido: 25-03-17 Revisado: $25-07-17$ Aceptado: $12-09-17$ Publicado: 11-10-17
RESUMEN. El análisis de los estilos de aprendizaje en estudiantes universitarios, considerando la carrera en la cual se encuentran, ha permitido establecer algunas predicciones sobre sus rendimientos académicos. El presente estudio tiene como objetivo principal identificar y establecer relaciones entre los estilos de aprendizaje, el promedio de bachillerato y el perfil de la carrera por cursar, en dos cohortes de estudiantes de la Licenciatura en Comunicación-Humana de la UAEM. Se aplicó el cuestionario de estilos de aprendizaje CHAEA a 166 estudiantes de nuevo ingreso de las cohortes 2015 y 2016, que cursaban el propedéutico para la carrera en Comunicación Humana, quienes además proporcionaron su promedio de ingreso y si habían sido reubicados. Los resultados reflejan diferencias significativas en los dominios de los estilos de aprendizaje en las dos cohortes, no existen diferencias entre el promedio de bachillerato entre ellas, en la cohorte 2016 se encontró una correlación negativa moderada del promedio de bachillerato con el estilo activo, a mayor dominio de estilo activo, menor promedio. Los estilos dominantes en las cohortes fueron el activo y pragmático, correspondiendo al perfil curricular de la carrera donde el incremento de horas prácticas sobre las teóricas se presenta de manera significativa en cada semestre.
Palabras clave: estilos de aprendizaje,

CHAEA,

promedio escolar, elección

de carrera universitarios

Citar como: Martínez-Flores, F. G. \& Delgado-Sánchez, U. (2017). Estilos de aprendizaje, elección de carrera y perfil curricular en estudiantes de Comunicación Humana. Revista Digital de Investigación en Docencia Universitaria, 11(2), 274-287. doi: http://dx.doi.org/10.19083/ridu.11.526

*E-mail: maga.mtzfagmail.com **E-mail: ulises.delgadođuaem.mx 
ABSTRACT. The analysis of the learning styles of university students, considering the study program in which they are enrolled, has allowed to make some predictions regarding their academic performance. This study is aimed at identifying and establishing relationships between learning styles, the average grade obtained in high school, and the study program profile, in two cohorts of students of the Human Communication Program at the UAEM. The CHAEA Learning Styles Questionnaire was applied to 166 newly admitted students of the 2015 and 2016 cohorts who were taking the induction course for the Human Communication Study program. These students had also provided their admittance average and information on whether they had been placed on a more suitable degree program. The results reflect significant differences in learning styles in both cohorts; there are no differences between the high school average between them; in the 2016 cohort, a moderate negative correlation was found between high school average and active style: the higher the active style, the lower the average. The dominant styles in the cohorts were active and pragmatic, which corresponds to the curricular profile of the study program, where an increase of practice hours over theory hours is more significant each semester.

RESUMO. A análise dos estilos de aprendizagem em estudantes universitários, considerando o curso no qual se encontram, permitiu estabelecer algumas previsões sobre seu desempenho acadêmico. O presente estudo tem como objetivo principal identificar e estabelecer relações entre os estilos de aprendizagem, a média do ensino secundário e o perfil do curso por estudar, em duas turmas de alunos do Curso de Comunicação Humana da UAEM. O questionário de estilos de aprendizagem CHAEA foi aplicado a 166 estudantes recém-matriculados das turmas de 2015 e 2016, que estudavam na preparatória para o curso de Comunicação Humana, eles também forneceram sua renda média e se haviam sido transferidos. Os resultados refletem diferenças significativas nos domínios dos estilos de aprendizagem nas duas turmas, não há diferença entre a média de ensino secundário entre eles, na turma de 2016, uma correlação negativa moderada foi encontrada entre a média do ensino secundário e o estilo ativo, ao maior Domínio de estilo ativo, menor média. Os estilos dominantes nas turmas foram o ativo e pragmático, correspondentes ao perfil curricular do curso onde o aumento das horas práticas sobre as teóricas se apresenta de forma significativa em cada semestre.

Palavraschave: estilos de aprendizagem, CHAEA, média escolar, escolha do curso universitário. 
La tendencia en el análisis de los fenómenos educativos a nivel universitario ha transitado de los estudios centrados en el desempeño académico del estudiante, a la exploración de las múltiples variables vinculadas con los factores contextuales y personales de los estudiantes que pueden influir en su rezago, deserción, y fracaso académico. Factores como los relativos a la institución de procedencia (promedio y tipo de bachillerato); a la institución receptora (calificación en el examen de admisión y área de conocimientol, así como el nivel escolar de los padres y características socioeconómicas (Arias \& Flores, 2005; Arias, Chávez \& Muñoz, 2006; Cu-Balan, 2005; Duarte \& Galaz, 2006; Olmos \& Olmos, 2011), han tomado mayor relevancia para los investigadores que en el pasado. Con respecto al análisis del aprendizaje en la universidad, ha cobrado fuerza una línea de investigación que se centra en el estudiante, tratando de identificar sus estilos y estrategias de aprendizaje, el tiempo que dedica al estudio, su motivación, la elección de carrera, la percepción que tienen los propios estudiantes sobre sus habilidades, la actividad laboral paralela, competencias cognitivas, entre otros (Bazán \& Soto, 2013; Delgado, Cárdenas, Flores \& Guzmán, 2014; Gravini \& Iriarte, 2008; Juárez, Rodríguez \& Luna, 2012; Villar, Vieira, Hernández \& Nunes, 2012; Zandonemi, Pacífico, Pagura, Canale \& Nessier, 2011).

Respecto a las variables atribuidas al estudiante, se han realizado numerosas investigaciones que se enfocan a los estilos de aprendizaje; este término fue definido a finales de la década de los ochenta por Keefe (1988), quien los concebía como los rasgos cognitivos, afectivos y fisiológicos, que sirven como indicadores relativamente estables, de cómo los discentes perciben, interaccionan y responden a sus ambientes de aprendizaje; por extensión, Camarero, Martín y Herrero (2000) declaran que los estilos de aprendizaje son un recurso que permite explicar las diferentes forma de abordar, planificar y responder ante las demandas de aprendizaje.

La evaluación de los estilos de aprendizaje, a pesar de su importancia, no goza de los consensos necesarios para generar un conocimiento uniforme acerca de ellos. En la actualidad existen diferentes modelos de evaluación de los mismos, como el propuesto por Rita Dunn y Kenneth Dunn con el Learning Styles Inventory (LSI), donde identifican aspectos relacionados con el ambiente inmediato, la propia emotividad del estudiante, las necesidades sociológicas, necesidades físicas y las necesidades psicológicas (Gallego \& Alonso, 2012; Dunn, 1984); la propuesta de Felder y Silverman con el Index of Learning Styles (ILS) el cual identifica a los estilos en cuanto a la percepción, representación, procesamiento y comprensión (Felder \& Spurlin, 2005); la propuesta de Kolb con el Learning Style Inventory (LSI) que clasifica a los estilos en convergente, divergente, asimilador y acomodador (Cabrera, 2004; Kolb, 1981; Page, 2006); y la propuesta de Honey-Alonso con el Cuestionario de Honey-Alonso de Estilos de Aprendizaje (CHAEA) que identifica cuatro estilos de aprendizaje que son el activo, reflexivo, teórico y pragmático (Esguerra \& Guerrero, 2010). Esta última, es la opción de evaluación de los estilos de aprendizaje de mayor uso en Iberoamérica.

El CHAEA es una prueba autoadministrable con puntuación dicotómica, de acuerdo (signo +) o en desacuerdo (signo -). Cuenta con 80 afirmaciones divididas en cuatro secciones de 20 ítems correspondientes a los cuatro estilos de aprendizaje, que son: activo, reflexivo, teórico y pragmático. Estos describen brevemente a continuación. 
- Estilo Activo: las personas con este estilo se involucran de manera plena y sin prejuicios en experiencias nuevas, son intolerantes a plazos prolongados, prefieren los resultados inmediatos. Son de mente abierta, con un nivel bajo de escepticismo. Acometen con entusiasmo las tareas novedosas, les gusta trabajar en grupo siendo empáticos con los demás. Para ellos aprender es encontrar la respuesta a la pregunta: ¿cómo?

- Estilo reflexivo: las personas con este estilo tienden a considerar sus experiencias y oportunidades desde diferentes perspectivas, reúnen datos, los analizan meticulosamente para llegar a conclusiones sólidas. Estas personas consideran todas las alternativas posibles antes de emprender una tarea. Analizan cómo actúan los demás, y no intervienen hasta que se han adueñado de la situación. Para ellos aprender es encontrar la respuesta a la pregunta: ¿por qué?

- Estilo teórico: son personas que proceden por etapas lógicas, por lo que ajustan sus observaciones y experiencias a teorías lógicas y complejas. Tienden a analizar, sintetizar, buscar la racionalidad y la objetividad en todo. Para ellos aprender es encontrar la respuesta a la pregunta: ¿qué?

- Estilo pragmático: son personas que les gusta la aplicación práctica de las ideas y su experimentación, por lo que suelen ser impacientes ante la teorización. Les gusta actuar rápidamente y con seguridad con aquellas ideas y proyectos que les atraen por lo que toman decisiones con rapidez y gustan de resolver problemas. Para ellos aprender es encontrar la respuesta a la pregunta: ¿qué pasaría? (Alonso, Gallego \& Honey, 1999; Aragón \& Jiménez, 2009; Gallego \& Alonso, 2012).

Entre las investigaciones realizadas en Iberoamérica con este instrumento destaca el estudio de Camarero et al. (2000) con estudiantes de diferentes disciplinas, de la Universidad de Oviedo, en el cual identificaron que los alumnos de Informática, Física, Matemáticas, Derecho y Magisterio muestran un predominante estilo reflexivo; y en menor grado presentan los estilos teórico, activo y pragmático. Paralelamente, Alonso et al. (1999) encontraron correspondencias entre los estilos de aprendizaje y las carreras cursadas en varias universidades españolas, por ejemplo, en las carreras técnicas (Aeronáutica, Caminos, Agronomía, Industrial, Informática, Telecomunicaciones) predomina el estilo pragmático, seguido de los estilos activo, reflexivo y teórico; en las Facultades de Humanidades (Filosofía y Ciencias de la Educación, Psicología, Filología, Geografía e Historia, Derecho, Política y Sociología, Economía, Ciencias de la Información, Formación del Profesorado) desarrollan principalmente el estilo activo, seguido del reflexivo, teórico y pragmático; y las Facultades Experimentales (Química, Física, Matemáticas, Medicina, Farmacia, Veterinaria, Enfermeríal tienen dominio principalmente de estilo reflexivo y teórico.

Morales, Hidalgo, García y Molinar (2013) presentaron hallazgos donde estudiantes de las carreras de Ingeniería en Computación, y las licenciaturas en Administración, en Contaduría, en Derecho, Informática Administrativa y en Psicología, usan en menor grado el estilo activo, siendo el de mayor presencia el reflexivo, a excepción de la Licenciatura en Derecho que identifica mayormente el estilo teórico. Coincidiendo con los resultados anteriores, De la Paz, Bautista, Mendoza y De la Paz (2012) encontraron que tanto los estudiantes de Ingeniería Química, Química Farmacéutica Biológica, y Biología, presentaron un dominio mayor del estilo reflexivo y menor del activo. Adicionalmente, el 
estudio de Loret de Mola (2008) realizado en el Perú converge con los hallazgos de las investigaciones anteriores en el predominio del uso reflexivo en estudiantes del Instituto Superior Pedagógico Privado, seguido de los estilos teórico, activo y pragmático. Este estilo correlacionó de manera positiva con el desempeño académico.

En un estudio realizado en Costa Rica con estudiantes de la licenciatura de Rendimiento Deportivo se encontró mayor presencia del estilo reflexivo, seguido del teórico, pragmático y activo. Por su parte, Salas-Cabrera (2014) encontró en una muestra de 242 estudiantes de Bacteriología y Laboratorio Clínico que el estilo predominante para promedio alto, medio y bajo correspondió al reflexivo. Más recientemente, Delgado, Martínez, Moreno y Padilla (2016) a diferencia de los estudios anteriores encontraron que tanto los estudiantes de Psicología como los de Maestría en Educación Preescolar tienen predominio del estilo activo y menor uso del estilo reflexivo.

Diversos resultados hacen notable la relación entre los estilos de aprendizaje y variables, tales como el promedio acumulado de bachillerato y el promedio acumulado de la carrera. Por ejemplo, Morales, et al. (2013) encontraron que el estilo activo correlacionó positivamente con el promedio de bachillerato en una población de 336 estudiantes de bachillerato. En otro estudio, Esguerra y Guerrero (2010) con 159 estudiantes de psicología no encontraron correlaciones estadísticamente significativas entre los estilos de aprendizaje y el promedio acumulado en diferentes semestres de la carrera. Ruiz, Trillos y Morales (2006) aplicaron el CHAEA a 101 estudiantes de la Universidad Tecnológica de Bolivia y encontraron una correlación positiva y significativa entre el rendimiento académico con el estilo teórico y estilo reflexivo. Por otra parte, Blumen, Rivero y Guerrero (2011) también hallaron una correlación positiva moderada entre el rendimiento académico y el estilo teórico. De igual manera, Delgado, Martínez y Flores (2016) aplicaron el cuestionario CHAEA a 300 estudiantes de Psicología de Universidad Autónoma del Estado de Morelos (UAEM) y a 336 estudiantes de psicología de la Universidad Nacional Autónoma de México, FES Iztacala (UNAM), encontraron una convergencia en los estilos de aprendizaje de ambas universidades, presentando únicamente los estudiantes de UNAM diferencias significativas en el estilo teórico con un puntaje de 7.9 contra 7.4 de la UAEM.

Con el objetivo de evaluar de manera fina el desarrollo de los estilos de aprendizaje, varios investigadores se han orientado al análisis de diferentes cohortes generacionales al interior de un número limitado de carreras. Solís y Arcudia (2010) aplicaron el Index of Learning Styles (ILS) a 364 estudiantes de la carrera de ingeniería civil, identificando que en ninguna de las dimensiones se encontró diferencia significativa entre los grupos de estudiantes que ingresaron en cuatro cohortes anuales en la preferencia de estilos de aprendizaje. Ventura y Angelione (2014) no encontraron diferencias estadísticamente significativas en la cohorte de estudiantes de ingeniería de 2012 y 2013 , en el dominio de sus estilos de aprendizaje. Al estudiar cohortes generacionales en educación superior es necesario considerar las condiciones de ingreso a la universidad, esto es, si el estudiante logró ingresar a su primera opción o si es reubicado en una carrera diferente por la institución. En torno a ello, Fuentes (2010) expresa que la elección inadecuada de la carrera universitaria por estudiar es una situación que muy frecuentemente presentan los jóvenes y que constituye uno de los principales factores que tiene como consecuencia la deserción universitaria. 
En un estudio realizado con 749 estudiantes de la Benemérita Universidad Autónoma de Puebla (BUAP), en México, en relación con el abandono de la carrera, 382 estudiantes refirieron que fue por motivos de ingreso a una actividad laboral, 237 por estudiar otra carrera en la misma BUAP y 73 por estudiar en otra universidad principalmente; afirmando el $19.9 \%$ de la población en estudio que la carrera en la que estaban no era su vocación, el 13.4\% que no le agrado el perfil de la carrera (Vries, León, Romero \& Hernández, 2011). Por ello Alonso et al. (1999) así como Becher y Trowler (2001; como se citó en Ventura, 2013) afirman que en el área disciplinar que cada carrera posee, cada comunidad académica tiene tradiciones, categorías de pensamiento y una determinada identidad asociada a ciertos códigos, prácticas y perfiles típicos; complementando Ventura (2013), quien estableció que el aprendizaje de una disciplina universitaria involucra no solo la apropiación de conceptos, sino también la adquisición de ciertas habilidades y estilos de aprendizaje, puesto que la formación en un campo disciplinar puede incidir en el aprendizaje de ciertos estilos idiosincráticos.

En México, el Consejo para la Acreditación de la Educación Superior, Asociación. Civil ICOPAES, 2016), en su marco de referencia del 2012 considera como categoría de análisis el personal académico, el estudiante, el plan de estudios, la evaluación del aprendizaje, la formación integral, el servicio de apoyo para el aprendizaje, la vinculación-extensión, la investigación, la infraestructura y equipamiento, la gestión administrativa y financiamiento. Las categorías ligadas al estudiante no destacan de manera relevante la necesidad de un análisis del ajuste de sus estilos personales al perfil curricular de la carrera estudiada, lo cual se constituye en un área de oportunidad para la investigación educativa.

Una carrera que recientemente ha crecido en demanda en la Universidad Autónoma de Morelos (UAEM), es la Licenciatura en Comunicación Humana (LCH), la cual pertenece a las Ciencias de la Salud y del Comportamiento, y en donde se han realizado reubicaciones, entendiéndose estas como la posibilidad de estudiantes de ingresar a otra carrera que no fue su primera opción, siempre y cuando en su examen de admisión tengan un puntaje de 32.50 (UAEM, 2017). El mapa curricular de esta se divide en cinco ejes de formación (lenguaje y audición, biomédico, psicopedagógico, social y metodológico), y dos ejes de asignaturas (prácticas y clínicas, y optativas); así mismo, consta de tres etapas de formación que son la básica, la disciplinar y la profesionalizante. En este mapa curricular se estipula la continuidad de ciertas materias, así como la carga de horas teóricas y prácticas que tienen cada una de ellas. Con base en lo anterior, la presente investigación tiene por objetivos: 1) identificar si existen diferencias en los estilos de aprendizaje que presentan los estudiantes que cursan el propedéutico de la LCH en los cohortes 2015 y 2016; 2) identificar si existen diferencias significativas entre el estilo de aprendizaje identificado y el promedio acumulado obtenido en el grado inmediato anterior (bachillerato) en las dos cohortes; 3) estimar si existe alguna correlación entre el promedio acumulado y los estilos de aprendizaje en las dos cohortes; 4) identificar acorde con el mapa curricular de la LCH cuál sería el estilo que curricularmente predomina en ésta formación. Como predicción hipotética se plantea que los estudiantes de las cohortes diferentes tendrán similar dominio en las dimensiones de los estilos de aprendizaje; y que puede existir una correlación positiva entre el estilo reflexivo y el promedio. 


\section{Tipo de investigación}

\section{MÉTODO}

Estudio transversal con dos cohortes independientes.

\section{Participantes}

166 estudiantes de la LCH de la UAEM. 78 estudiantes de la cohorte 2015 (70 mujeres y 8 hombres, con un rango de edad de 17 a 27 años) y 92 estudiantes de la cohorte 2016 (78 mujeres y 14 hombres, con un rango de 17 a 31 años) inscritos en el curso propedéutico que ofrece LCH. Cada cohorte comprende la población total de estudiantes por generación, por lo que no fue necesario llevar a cabo procedimientos de muestreo. Únicamente en la cohorte 2016, hubo un $31.86 \%$ de estudiantes reubicados, que aspiraban al ingresar a las siguientes carreras: 11 a medicina, 6 a psicología, 6 a nutrición, 3 a derecho, 1 a enfermería, 1 a relaciones internacionales, y 1 a terapia física.

\section{Instrumentos}

Cuestionario Honey-Alonso de Estilos de Aprendizaje (CHAEA) en una versión reducida de 56 ítems dicotómicos, 13 ítems para el estilo activo ( $\mathrm{a}=.53$ ), 16 para el estilo pragmático ( $\mathrm{a}=.53$ ), 12 ítems para el estilo reflexivo $(a=.54)$ y 15 ítems para el estilo teórico $(a=.60)$, con un alfa total del instrumento de .0.67. Para la creación de esta versión validada para población mexicana se realizó un análisis factorial confirmatorio de primer orden con un total de 940 estudiantes de tres universidades mexicanas. A continuación se presentan los índices de ajuste de cada una de las dimensiones en su versión reducida. El estilo activo presentó un $\mathrm{CFI}=0.94$ y un $\mathrm{RMSEA}=0.03$, el estilo pragmático presentó un $\mathrm{CFI}=0.97$ y un RMSEA $=0.02$, el estilo reflexivo un $C F I=0.98$ y un $R M S E A=0.02$ y el estilo teórico presentó un $C F I=0.92$ y un RMSEA=0.03. El índice RMSEA es considerado óptimo cuando sus valores son inferiores a 0.06 ( Hu \& Bentler, 1995), y el CFI si presenta valores de 0.90 o superiores (Cea, 2004).

\section{Procedimiento}

Se acudió a los salones donde se impartían los cursos propedéuticos en cada cohorte, se informó a los estudiantes sobre los objetivos del estudio y se les solicitó su consentimiento por escrito para participar de manera voluntaria en la investigación. Dado su consentimiento se les pidió que especificaran su promedio de calificaciones obtenido en el nivel inmediato anterior y que contestaran el cuestionario CHAEA. Después de aplicar el Cuestionario de estilos de aprendizaje y de contabilizar las horas teóricas y prácticas del plan de estudios de la licenciatura en Comunicación Humana fueron vaciados los datos a una base en SPSS, versión 22, para correr posteriormente los estadísticos necesarios. Para los estilos de aprendizaje se realizaron ANOVA de un factor y correlaciones, utilizándose para la comparación de las horas teóricas y prácticas del mapa curricular una comparación por medio de la prueba de rangos señalados de Wilcoxon.

\section{RESULTADOS}

A partir del promedio de respuestas positivas a los reactivos de cada uno de los estilos de aprendizaje se encontró que los estudiantes de la cohorte 2015 presentaron una predominancia del estilo activo (6.01), seguido por el uso del estilo teórico (5.68), pragmático (5.45) y reflexivo (2.89) respectivamente; mientras que los estudiantes de la cohorte 2016 presentaron un predominio del estilo pragmático 
(10.61), seguido del teórico (9.49), reflexivo (9.07) y activo (6.92). La dispersión de ambas cohortes no se aproxima a los valores de la dispersión ideal, pudiendo considerarse que los estilos en la cohorte 2015 muy bajos, y los de la cohorte 2016 bajos, como puede apreciarse en la figura 1.

\section{ESTILOS DE APRENDIZAJE EN ESTUDIANTES DEL PROPEDÉUTICO DE LCH}

IDEAL

Pragmático

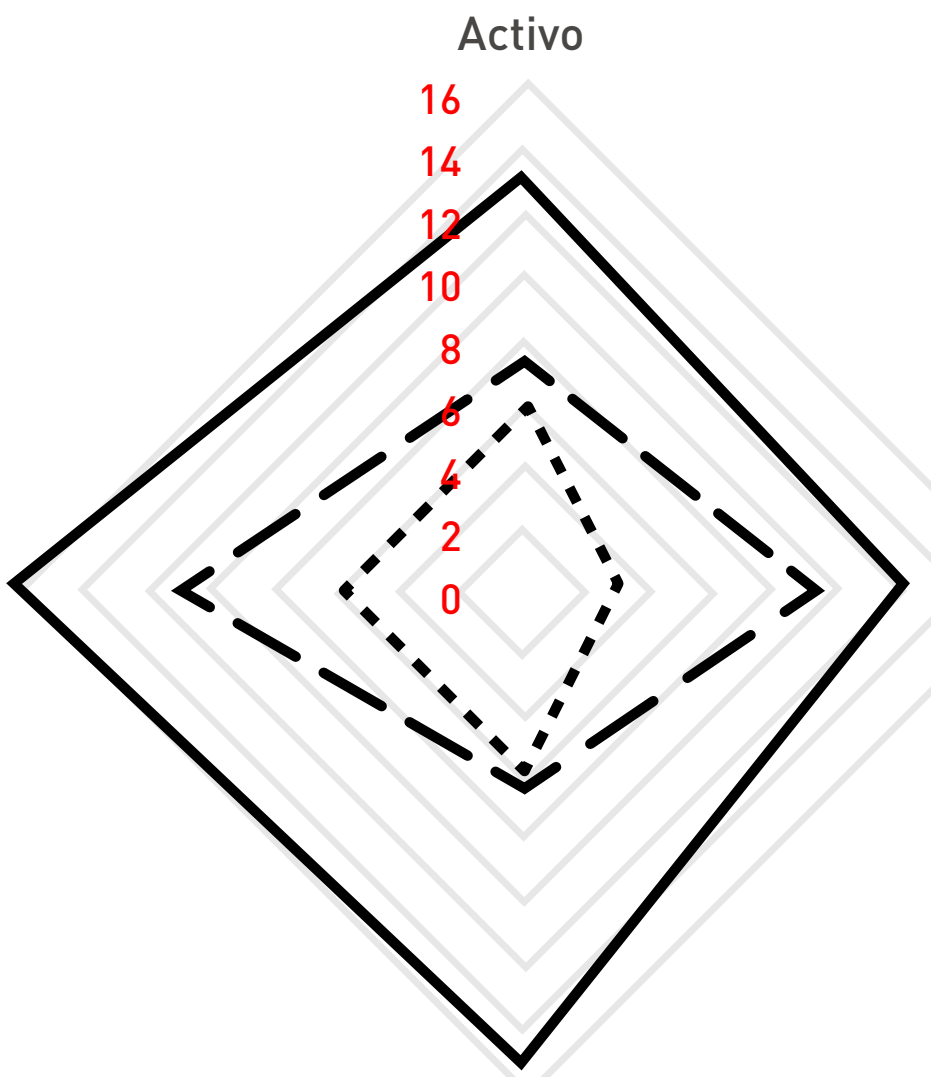

Reflexivo

Teórico

Figura 1. Dispersión de los estilos de aprendizaje en dos cohortes generacionales de la FCH.

El análisis de las diferencias entre ambas cohortes denota diferencias significativas entre las dos cohortes en los cuatro estilos de aprendizaje (ver tabla1). La cohorte 2016 presentó un $15.14 \%$ más en el uso del estilo activo que la cohorte 2015 , así mismo un $67.07 \%$ más en el dominio del estilo teórico, un $94.67 \%$ más del estilo pragmático, y un $213.84 \%$ en el estilo reflexivo.

Acorde con la tabla 2, la cohorte 2015 presentó un uso del estilo activo en rango moderado, el estilo teórico y pragmático están en el rango bajo y el estilo reflexivo en el rango muy bajo; por otra parte, la cohorte 2016 presentó un rango del estilo activo moderado, el estilo pragmático un rango alto, de los estilos reflexivo y teórico un uso moderado. 
Tabla 1

Estilos de aprendizaje en dos cohortes

\begin{tabular}{|c|c|c|c|c|c|c|}
\hline \multirow{2}{*}{ Estilo de aprendizaje } & \multicolumn{2}{|c|}{ Cohorte 2015} & \multicolumn{2}{|c|}{ Cohorte 2016} & \multirow{2}{*}{$t$} & \multirow{2}{*}{$p$} \\
\hline & $M$ & DT & M & DT & & \\
\hline Estilo Activo & 6.01 & 2.29 & 6.92 & 2.32 & -2.52 & .012 \\
\hline Estilo pragmático & 5.45 & 2.03 & 10.61 & 2.35 & -14.87 & .000 \\
\hline Estilo reflexivo & 2.89 & 1.99 & 9.07 & 1.80 & -20.90 & .000 \\
\hline Estilo teórico & 5.68 & 2.71 & 9.49 & 2.6 & -9.27 & .000 \\
\hline Promedio de bachillerato & 8.4 & .66 & 8.24 & .71 & 1.49 & .13 \\
\hline
\end{tabular}

\section{Tabla 2}

Rangos de los estilos de aprendizaje

\begin{tabular}{|l|c|c|c|c|c|}
\hline Estilo & Muy bajo & Bajo & Moderado & Alta & Muy alta \\
\hline Activo & $1-2$ & $3-4$ & $5-7$ & $8-10$ & $11-13$ \\
Pragmático & $1-3$ & $4-6$ & $7-9$ & $10-12$ & $13-16$ \\
Reflexivo & $1-2$ & $3-4$ & $5-6$ & $7-9$ & $10-12$ \\
Teórico & $1-3$ & $4-6$ & $7-9$ & $10-12$ & $13-15$ \\
\hline
\end{tabular}

Los hallazgos encontrados a partir del ANOVA de un factor, reflejan en la cohorte 2015 que no existe una relación significativa del estilo activo $\mathrm{F}_{(27,71)}=.75, p=.78$, el estilo pragmático $\mathrm{F}_{(27,70)}=.79, p=.74$, el estilo reflexivo $\mathrm{F}_{(26,70)}=.79, p=.73$ y el estilo teórico $\mathrm{F}_{(27,72)}=.88, p=.63$ con el desempeño académico del nivel inmediato inferior. Esto mismo sucede en la cohorte 2016 , estilo activo $F_{(37,86)}=.94, p=.57$, el estilo pragmático $\mathrm{F}_{(36,85)}=1.24, p=.23$, el estilo reflexivo $\mathrm{F}_{(37,85)}=.73, p=.83$ y estilo teórico $\mathrm{F}_{(36,86)}=.87, p=.66$.

No se encontraron correlaciones estadísticamente significativas entre el promedio y el estilo activo rho $=.02, p=.87$, pragmático rho $=-.1, p=.43$, reflexivo rho $=-.22, p=.06$ y teórico $r h o=-.03, p=.81$ en la cohorte 2015. En la cohorte 2016 no se encontró una correlación significativa del promedio con los estilos pragmático rho $=-.15, p=.16$, el estilo reflexivo $r h o=.001, p=.99$ y el estilo teórico rho $=.05, p=.63$, encontrándose una correlación negativa débil con el estilo activo rho= $-.31, p=.004$.

Un análisis paralelo del mapa curricular de la $\mathrm{LCH}$, que proporciona un indicador indirecto del tipo de demandas académicas potenciales para los estudiantes de esta carrera, permite identificar que, a través de nueve semestres, cada materia tiene horas asignadas a los contenidos teóricos y horas que consideran los aspectos prácticos (ver tabla3), siendo notable que, al pasar los periodos incrementan las horas prácticas disminuyendo las horas teóricas. 
Tabla 3

Rangos de los estilos de aprendizaje

\begin{tabular}{|c|c|c|c|c|c|c|}
\hline Periodo & $\begin{array}{l}\text { Total de } \\
\text { materias }\end{array}$ & $\begin{array}{c}\text { Total de } \\
\text { horas }\end{array}$ & $\begin{array}{l}\text { Horas } \\
\text { teóricas }\end{array}$ & $\begin{array}{c}\text { Porcentaje } \\
\text { de horas } \\
\text { teóricas }\end{array}$ & $\begin{array}{c}\text { Horas } \\
\text { prácticas }\end{array}$ & $\begin{array}{c}\text { Porcentaje } \\
\text { de horas } \\
\text { prácticas }\end{array}$ \\
\hline $1^{\circ}$ & 6 & 24 & 12 & $50 \%$ & 12 & $50 \%$ \\
\hline $2^{\circ}$ & 8 & 36 & 15 & $41.66 \%$ & 21 & 58.33 \\
\hline $3^{\circ}$ & 7 & 32 & 13 & $40.62 \%$ & 19 & $59.37 \%$ \\
\hline $4^{\circ}$ & 8 & 35 & 14 & $40 \%$ & 21 & $60 \%$ \\
\hline $5^{\circ}$ & 7 & 38 & 12 & $31.57 \%$ & 26 & $68.42 \%$ \\
\hline $6^{\circ}$ & 7 & 36 & 12 & $33.33 \%$ & 24 & $66.66 \%$ \\
\hline $7^{\circ}$ & 6 & 36 & 10 & $27.77 \%$ & 26 & $72.22 \%$ \\
\hline $8^{\circ}$ & 4 & 28 & 6 & $21.42 \%$ & 22 & $78.57 \%$ \\
\hline $9^{\circ}$ & 1 & 35 & 0 & $0 \%$ & 35 & $100 \%$ \\
\hline
\end{tabular}

Se encontraron diferencias significativas $p=.012$ entre la cantidad de horas teóricas y horas prácticas que tiene el currículo de la Licenciatura de Comunicación Humana, siendo las de mayor dominancia las prácticas.

\section{DISCUSIÓN}

Con relación al primer objetivo, identificar los estilos de aprendizaje presentes en los estudiantes que cursan el propedéutico de la Licenciatura de Comunicación Humana en 2015 y 2016, se encontró que los estudiantes de 2015 tienen una predominancia mayor al estilo activo, de lo cual se infiere que son estudiantes a los que les interesan las experiencias nuevas, les gustan los resultados inmediatos, el debate, el diálogo y les gusta trabajar en grupo; en contraparte, el estilo reflexivo es el de menor uso, siendo poca la tendencia a considerar sus experiencias y observarlas desde diferentes perspectivas, reunir datos, analizarlos con detenimiento para llegar a conclusiones. Por lo que los hallazgos encontrados destacan que los estudiantes del curso propedéutico presentaron mayor dominio del estilo activo, siendo coincidente con Alonso et al. (1999), quienes destacan que las carreras que pertenecen a Humanidades tienen mayor dominio de este estilo, coincidiendo con los resultados de la investigación de Delgado et al. (2016) con estudiantes de Psicología, donde el estilo con menos presencia en ambas poblaciones es el reflexivo y el de mayor es el activo; siendo importante destacar que aun y cuando su dominio mayor es el estilo activo, este se encuentra en un nivel moderado, estando por encima de los otros que están en un dominio bajo o muy bajos. Si bien la cohorte 2016 presentó un mayor puntaje en el uso del estilo activo que la cohorte 2015, este estilo no fue el que predominó, sino el pragmático, encontrándose este estilo en un dominio alto, siendo personas que les gusta la aplicación práctica de las ideas y su experimentación, teniendo fortalezas al estar los tres estilos restantes en un dominio moderado, siendo esta cohorte la que presenta un dominio de los estilos de aprendizaje 
mayor. La predominancia del estilo activo y pragmático en estas dos cohortes, está en concordancia con las necesidades del mapa curricular donde existe mayor dominancia de manera exponencial periodo a periodo de horas prácticas donde los estudiantes tienen que aplicar el conocimiento aprendido en clase y resolver problemáticas vinculados con los temas; tanto el dominio del estilo activo como del estilo pragmático son fundamentales como competencias en los estudiantes de Comunicación Humana, esto por la cantidad de horas prácticas que tienen, debido a que desde el tercer semestre tienen trabajo de campo, ya sea en ambientes clínicos o educativos, realizando entrevistas a padres, evaluaciones, diagnósticos e intervenciones tanto en niños como en adultos, siendo necesario en estos espacios actuar de una manera efectiva y eficiente ante diferentes situaciones y contingencias. Estos resultados contrastan con los encontrados por Loret de Mola (2008) en estudiantes del Instituto Superior Pedagógico, donde el dominio preferente fue sobre el estilo reflexivo y los hallazgos de Morales et al. (2013) quienes encontraron mayor dominio del estilo reflexivo en Ingeniería en Computación, Licenciatura en Administración, Licenciatura en Contaduría, Licenciatura en Derecho, Licenciatura en Informática Administrativa y Licenciatura en Psicología. Esto permite identificar que existen diferencias entre el tipo de carrera.

A diferencia de los resultados que correlacionan el estilo reflexivo con el rendimiento académico (Blanco, 2014; Loret de Mola, 2008), en el presente estudio se encontró que solo la cohorte 2016 correlaciona negativa y débilmente con el estilo activo, por lo que a mayor dominio de estilo activo menor promedio de bachillerato, esto puede deberse a que en esta etapa escolar se priorizan las materias teóricas sobre las prácticas; la cohorte 2015 es coincidente con los resultados encontrados por Esguerra y Guerrero (2010) en estudiantes de psicología donde no se encontraron diferencias significativas entre el promedio y los estilos de aprendizaje.

En cuanto a identificar acorde con el mapa curricular de la Licenciatura en Comunicación Humana cuál sería el estilo que curricularmente predomina en ésta formación, como se mencionó con antelación los resultados según la descripción del estilo activo y pragmático dejan en evidencia que los estudiantes del curso propedéutico son estudiantes que les gusta el diálogo y el debate, les gusta trabajar en grupo, y la pregunta detonadora del aprendizaje en ellos es el ¿cómo?, así como les gusta aplicar las ideas y su experimentación, tomando decisiones con rapidez y resolviendo problemas, otra pregunta que también predomina es ¿qué pasaría sí? Esto evidencia que los estudiantes de las dos cohortes del curso propedéutico presentan un dominio de los estilos que se vinculan con el desarrollo curricular de la carrera, en cuanto a la relación de la cantidad de horas prácticas y teóricas, siendo el estilo activo y pragmático el que se podría decir que domina el mapa curricular por la cantidad de horas prácticas que van incrementando de manera significativa periodo a periodo. Con los resultados encontrados se rechaza la hipótesis nula y se acepta la alternativa: estudiantes de cohortes diferentes tendrán diferente dominio en las dimensiones de los estilos de aprendizaje, esto pudo ser influenciado por la elección de carrera, ya que más del 30\% de la cohorte 2016 es reubicada; de igual manera se rechaza la hipótesis nula de que existe una correlación positiva entre el estilo reflexivo y el promedio, encontrando una correlación negativa entre el promedio y el estilo activo. 


\section{CONCLUSIONES}

Cabe destacar que las posibles diferencias en el uso de los estilos de aprendizaje de la cohorte 2015 y 2016 puede deberse a que en la cohorte 2015 no se aceptaron estudiantes de reubicación y en la cohorte 2016 sí existieron reubicaciones, esto quiere decir que cuando un estudiante postula en la UAEM a una carrera de primera opción y no acredita el puntaje determinado para la carrera de elección, tiene la posibilidad de ser reubicado en una segunda carrera. Algunos alumnos de la cohorte 2016 fueron reubicados de medicina, enfermería, psicología, nutrición, derecho, siendo carreras de humanidades. Los postulantes a primera opción de las carreras de medicina, psicología y enfermería, son los que obtienen mayor puntaje en el examen de admisión, estas carreras tienen estándares altos para sus aspirantes, quedand o fuera de estas en muchas ocasiones estudiantes que tienen promedio aprobatorio satisfactorio en el examen, esto por la capacidad de aspirantes que pueden aceptar, por lo que los estudiantes que no son aceptados aún y con promedio buenos, se les da la opción de reubicación. Si bien se observa que estas dos cohortes son significativamente diferentes en el dominio de sus estilos de aprendizaje, falta investigar las trayectorias escolares de ellas, para poder identificar si los reubicados permanecen o se dan de baja en la universidad y el impacto del dominio de los estilos de aprendizaje en el desempeño académico.

Aún falta identificar cuáles podrían ser las variables que correlacionan de manera significativa con el desempeño de los estudiantes en sus trayectorias, como es la escuela de procedencia, hábitos de estudio, elección de carrera, estilo de aprendizaje, estrategias de aprendizaje, planeación de sus tiempos. Así mismo, en futuras investigaciones se puede hacer un estudio de las trayectorias académicas de estas cohortes y generar programas de fortalecimiento de las mismas. Siendo relevante hacer en un futuro una investigación del dominio de los estilos de aprendizaje en diversas carreras, para identificar la predominancia de estilos por la preferencia disciplinar, así como la pertinencia o no de las reubicaciones o el papel que jugarían las tutorías académicas en estos casos. 


\section{REFERENCIAS}

Alonso, C., Gallego, D. \& Honey, P. (1999). Estilos de aprendizaje. Procedimientos de diagnóstico y mejora. Universidad de Deusto: Mensajero.

Aragón, M. \& Jiménez, Y. (2009). Diagnóstico de los estilos de aprendizaje en los estudiantes: Estrategia docente para elevar la calidad educativa. Revista de Investigación Educativa, 9, 1-21. Recuperado de https://goo.gl/CW9pwg

Arias, L. \& Flores, A. (2005). La satisfacción de los estudiantes con su carrera y su relación con el promedio y el sexo. El caso de la carrera de Contaduría de la Universidad Veracruzana en Nogales, Veracruz. Hitos de Ciencias Económico Administrativas, 29(1), 9-14.

Arias, L., Chávez, A. \& Muñoz, I. (2006). El aprovechamiento previo y la escuela de procedencia como predictores del aprovechamiento futuro: un caso. Revista Enseñanza e Investigación en Psicología, 11(1), 5-22. Recuperado de https://goo.gl/YhWTnw

Bazán, A. \& Soto, Y. (2013). Análisis factorial comprobatorio y confirmatorio del inventario ampliado de factores de carrera en estudiantes de una universidad privada. En A. Bazán y C. Butto (Coord.), Psicología y Contextos Educativos (pp. 255-277). México: Horizontes Educativos.

Blanco, E. (2014). Estilos de aprendizaje y promedio académico en estudiantes de bacteriología y laboratorio clínico. Revista de Estilos de Aprendizaje, 7(13), 202-219. Recuperado de https://goo.gl/ySJv6r

Blumen, S., Rivero, C. \& Guerrero, D. (2011). Universitarios en educación a distancia: estilos de aprendizaje y rendimiento académico. Revista de Psicología. 29(2), 225-243.

Cabrera, J. (2004). Fundamentos de un sistema didáctico del inglés con fines específicos centrados en los estilos de aprendizaje (Tesis doctoral inédita). Universidad de Pinal del Río, Santiago de Cuba.

Camarero, F., Martín. F. \& Herrero. J. (2000). Estilos y estrategias de aprendizaje en estudiantes universitarios. Psicothema, 12(4). 612-622. Recuperado de https://goo.gl/v4zExB

Cea, M. (2004). Análisis multivariable. Teoría y práctica en la investigación social. México: Síntesis.

Consejo para la Acreditación de la Educación Superior (2016). Marco General de Referencia para los Procesos de Acreditación de Programas Académicos de Tipo Superior. Recuperado de https://goo.gl/fq9ALV

Cu-Balán, G. (2005). El impacto de procedencia del nivel medio superior en el desempeño de los alumnos en el nivel universitario. Revista Electrónica Iberoamericana sobre Calidad, Eficacia y Cambio en Educación, 3(1), 764-769. Recuperado de https://goo.gl/YMLd58

De la Paz, J., Bautista, C., Mendoza, M. \& De la Paz, C. (junio 2012). Estilos de aprendizaje de las carreras de la FES Zaragoza, UNAM, Campus II. Ponencia presentada en el V Congreso Mundial de Estilos de Aprendizaje. Santander, España.

Delgado, U., Martínez, F., \& Flores, A. (2016). Análisis de los estilos y estrategias de aprendizaje en estudiantes de psicología. Investigación y práctica en psicología del desarrollo, 2, 138-146. Recuperado de https://goo.gl/VtRBv3

Delgado, U., Cárdenas, K., Flores, C. \& Guzmán, M. (noviembre 2014). Disposiciones para el aprendizaje y logro académico en estudiantes universitarios. Ponencia presentada en el Congreso Iberoamericano de Ciencia, Tecnología, Innovación y Educación. Buenos Aires, Argentina. Recuperado de https://goo.gl/sHh6Kr

Delgado, U., Martínez, F., Moreno. A. \& Padilla. L. (septiembre 2016). Estilos de aprendizaje en estudiantes de Psicología UAEM y LEPTIC-UPN. Ponencia presentada en VI Congreso Estudiantil y primer congreso internacional de investigación en Psicología. Universidad Autónoma del Estado de México, México.

Duarte, M. \& Galaz, J. (junio 2006). Predictores del desempeño académico en primer año de universidad en una institución pública estatal. Ponencia presentada en el $6^{\circ}$ Congreso Internacional Retos y Expectativas de la Universidad: El Papel de la Universidad en la Transformación de la Sociedad, Puebla, México.

Dunn, R. (1984). Learning Style: State of the Science. Theory into Practice, 23(1), 10-19.

Esguerra, G. \& Guerrero, P. (2010). Estilos de aprendizaje y rendimiento académico en estudiantes de psicología. Diversitas: Perspectivas en Psicología, 6(6), 97-109. doi: http://dx.doi.org/10.15332/s1794-9998.2010.0001.07

Felder, R. \& Spurlin, J. (2005). Applications, Reliability and Validity of the Index of Learning Styles. International Journal of Engeneering Education, 21(1), 103-112. Recuperado de https://goo.gl/EigjFw

Fuentes, M. (2010). La orientación profesional para elegir fundamentadamente una ocupación: propuesta alternativa. Revista Mexicana de Psicología, 27(2), 237-246. Recuperado de https://goo.gl/1g9K3E

Gallego, D. \& Alonso, C. (2012). Los estilos de aprendizaje como una estrategia pedagógica del siglo XXI. Revista electrónica de Socioeconomía, Estadística e Informática, 1(1), 20-41. Recuperado de https://goo.gl/xCLGHY

Gravini, M. \& Iriarte, F. (2008). Procesos metacognitivos de estudiantes con diferentes estilos de aprendizaje. Psicología desde el Caribe/ Universidad del Norte, 22, 1-24. Recuperado de https://goo.gl/icCy5M 
Hu, L. \& Bentler, P. (1998). Fit índices in covariance structure modeling: Sensitivity to underparameterized model misspecification. Psychological Methods, 3(4), 424-453. doi: http://dx.doi.org/10.1037/1082-989X.3.4.424

Juárez, C., Rodríguez, G. \& Luna, E. (2012). El cuestionario de estilos de aprendizaje CHAEA y la escala de estrategias de aprendizaje ACRA como herramienta potencial para la tutoría académica. Revista Estilos de Aprendizaje, 10(10), 1-31. Recuperado de https://goo.gl/ZpEuki

Keefe, J. (1988). Profiling and Utilizing Learning Style. Virginia: NASSP.

Kolb, D. (1981). Learning styles and disciplinary differences. USA: The Modern American Collage.

Loret de Mola, M. (2008). Los estilos de aprendizaje de Honey-Alonso y el rendimiento académico en las áreas de formación general y formación profesional básica de los estudiantes del instituto superior pedagógico privado "nuestra señora de Guadalupe" de la provincia de Huancayo-Perú. Revista de los Estilos de Aprendizaje, 1(1), 201-213. Recuperado de https://goo.gl/dEvBoK

Morales, A., Hidalgo, L., García, R. \& Molinar. J. (2013). Relación entre los estilos de aprendizaje, rendimiento académico y otras variables relevantes. Revista de Estilos de Aprendizaje, 12(11), 1-16. Recuperado de https://goo.gl/TBG5wN

Olmos, D. \& Olmos, R. (2011). Perfil de ingreso de alumnos con buen desempeño académico en el primer año de estudios. El caso de la Escuela de Diseño de la Universidad de la Salle Bajío. Nova Scientia, 3(6), 95-120. Recuperado de https://goo.gl/GbsbMX

Page, V. (2006). Styles d'apprentissage et rendements académiques dans les formations en ligne. (Tesis doctoral inédita). Université de Montréal, Montreal, Canadá.

Ruiz, B., Trillos, J.\& Morales, J. (2006). Estilos de aprendizaje y rendimiento académico en estudiantes universitarios. Revista Galego-Portuguesa de Psicoloxía e Educación, 13(11), 441-457.

Salas-Cabrera, J. (2014). Estilos de aprendizaje en estudiantes de la Escuela de Ciencias del Movimiento Humano y Calidad de vida. Revista Electrónica Educare, 18(3), 159-171. doi: http://dx.doi.org/10.15359/ree.18-3.9

Solís, R., \& Arcudia, C. (2010). Estilos de aprendizaje de los estudiantes de ingeniería civil. Educación en ingeniería, 10, 24-36. Recuperado de https://goo.gl/QeeoC1

Universidad Autónoma del Estado de Morelos. (2017). Ofrece UAEM nuevos lugares para reubicación de aspirantes. Recuperado de https://goo. $\mathrm{gl} / \mathrm{cEPMr} 7$

Ventura, A. (2013). Estilos de aprendizaje y comunidades disciplinares desde un enfoque situado de la cognición. Revista Internacional de Psicología, 12(2), 1-29. Recuperado de https://goo.gl/KMLNkS

Ventura, A. \& Angelione. L. (2014). Estilos de aprendizaje y enseñanza en ingeniería: una propuesta de educación adaptativa para primer año. Educación en ingeniería, 9(8), 178-189. Recuperado de https://goo.gl/CsYmVV

Villar, A., Vieira, A., Hernández, F. \& Nunes, A. (2012). Más que abandono de estudios, trayectorias de reubicación universitaria. Aproximación comparada al caso español y portugués. Revista Lusófona de Edusão, 21, 139-162. Recuperado de https://goo.gl/t7iAbZ

Vries, W., León, P., Romero, J. \& Hernández, I. (2011). ¿Desertores o decepcionado? Distintas causas para abandonar los estudios universitarios. Revista de Educación Superior, 9(4), 29-50. Recuperado de https://goo.gl/dXfwvY

Zandonemi, N., Pacífico, A., Pagura, F., Canale, S. \& Nessier, A. (2011). Estudiantes universitarios avanzados que interrumpieron sus carreras: trazos de sus trayectorias académicas y laborales. Ciencias Económicas, 9(2), 11-30. Recuperado de https://goo.gl/Nnr8vK

(c) Los autores. Este artículo es publicado por la Revista Digital de Investigación en Docencia Universitaria del Área de Institutional Research and Effectiveness de la Dirección de Aseguramiento de la Calidad, Universidad Peruana de Ciencias Aplicadas. Este es un artículo de acceso abierto, distribuido bajo los términos de la LicenciaCreativeCommons Atribución-Compartirlgual 4.0 Internacional.l http://creativecommons.org/licenses/bysa/4.0/l, que permite el uso no comercial, distribución y reproducción en cualquier medio, siempre que la obra original sea debidamente citada. 